\title{
Boosting pigment epithelial-derived factor: a promising approach for the treatment of early portal hypertension
}

\author{
Umberto Vespasiani-Gentilucci, ${ }^{1}$ Krista Rombouts $^{2}$
}

Portal hypertension (PHT) is a heterogeneous clinical entity which develops in patients with cirrhosis. It is responsible for many of the complications that occur in cirrhosis, including gastroesophageal varices, hepatorenal syndrome, ascites, hepatic encephalopathy and hypersplenism. ${ }^{1}$ There are three principal factors responsible for the development of PHT, namely (1) purely mechanical obstruction resulting from hepatic fibrosis and regenerative nodules; (2) contraction of sinusoidal and perisinusoidal contractile cells due to an imbalance between intrahepatic vasoconstrictory and vasodilatory mediators and (3) splanchnic vasodilation and increased portal blood flow. ${ }^{1}$ Factors leading to the development of PHT include hypoxia, oxidative stress, inflammation and shear stress as potential mediators for the angiogenic response. Vascular Endothelial Growth factor (VEGF) is a key mediator in regulating the angiogenic switch in many pathological conditions, and is also probably a major player in the neoangiogenic process during the development of a hyperdynamic splanchnic circulation, and the formation of portalsystemic collateral vessels. ${ }^{2}$ This has led to many studies seeking to identify signalling pathways that interact with, or affect the formation and action of VEGF switching.

Mejias reports in this issue $e^{3}$ a new mechanism and therapeutic effect by targeting endogenous pigment epithelium-derived factor (PEDF) in bile duct-ligated portal hypertensive rats. PEDF belongs to the subgroup of the so-called non-inhibitory serpins that lack the protease inhibitory activity but which interacts with a variety of cellular components and signalling cascades. For this reason, PEDF is best characterised as a multifunctional protein with therapeutic effects in many diseases. ${ }^{4}$

\footnotetext{
${ }^{1}$ Internal Medicine and Hepatology, University Campus Bio-Medico of Rome, Rome, Italy; ${ }^{2}$ Institute for Liver \& Digestive Health, University College London (UCL), Royal Free Hospital, London, UK

Authors contributed equally.

Correspondence to Dr Krista Rombouts, Division of Medicine, Royal Free Hospital \& University College London, Institute for Liver \& Digestive Health, Rowland Hill Street, London NW3 2PF, UK; k.rombouts@ucl.ac.uk
}

The authors demonstrated that by employing an adenoviral-mediated gene transfer of PEDF, which enhanced the expression of PEDF, only resulted in inhibition of neoangiogenesis. This is important, since any drug used to treat or prevent PHT should ideally inhibit neoangiogenesis without affecting the normal vessels. Moreover, in this experimental approach, by applying prevention trials and intervention trials, authors showed to diminish (1) significantly liver fibrosis by upregulation of matrix metalloproteinase-2 (MMP-2), (2) portosystemic collateralisation and (3) portal pressure in bile duct-ligated rats. To translate these data to man, the authors investigated PEDF localisation in HCV-related cirrhosis, and observed that PEDF was predominantly overexpressed in regenerative micronodules, at the interface between liver parenchyma and highly neovascularised fibrous septa. These PEDF-positive regions also correlated with high expression levels of VEGF. This observation was further substantiated with in vivo data where the authors showed a spatio-temporal PEDF expression that followed the upregulation of VEGF in bile duct ligation (BDL)-ligated rats, $\mathrm{CCl}_{4}$-induced cirrhotic livers, and during neoangiogenesis in the mesentery. Thus, this observation demonstrates an important element in underlying the mechanism of PEDF therapy to counteract VEGF-related actions during neoangiogenesis. Of note, in the BDL experimental model, PEDF was particularly effective when administered during the early phase of liver disease (prevention trial), consistent with its capability to interfere, in the liver, with the fibrogenesis/angiogenesis couple and, in the mesentery, with the neovascular response at the time of its maximal expression (see figure 1). These data indicate that, if translated into clinical usage, such drugs would interfere with neoangiogenesis at an early stage of PHT.

At present, we have various drugs that target almost exclusively the splanchnic and intrahepatic vascular tone and which are used clinically to treat the complications of PHT. However, there are two major unmet clinical needs: namely to halt or prevent the rise of portal pressure before clinically significant PHT develops, and to increase efficacy, once PHT has developed, with new therapeutic targets. With regard to the first point, longitudinal studies in cirrhotic patients with baseline haemodynamic assessment have clearly demonstrated that all PHT complications do not develop until the hepatic venous pressure gradient (HVPG) exceeds $10 \mathrm{~mm} \mathrm{Hg}$, namely the range of clinically significant PHT. ${ }^{6}$ Therefore, a drug which is able to prevent an increase of portal

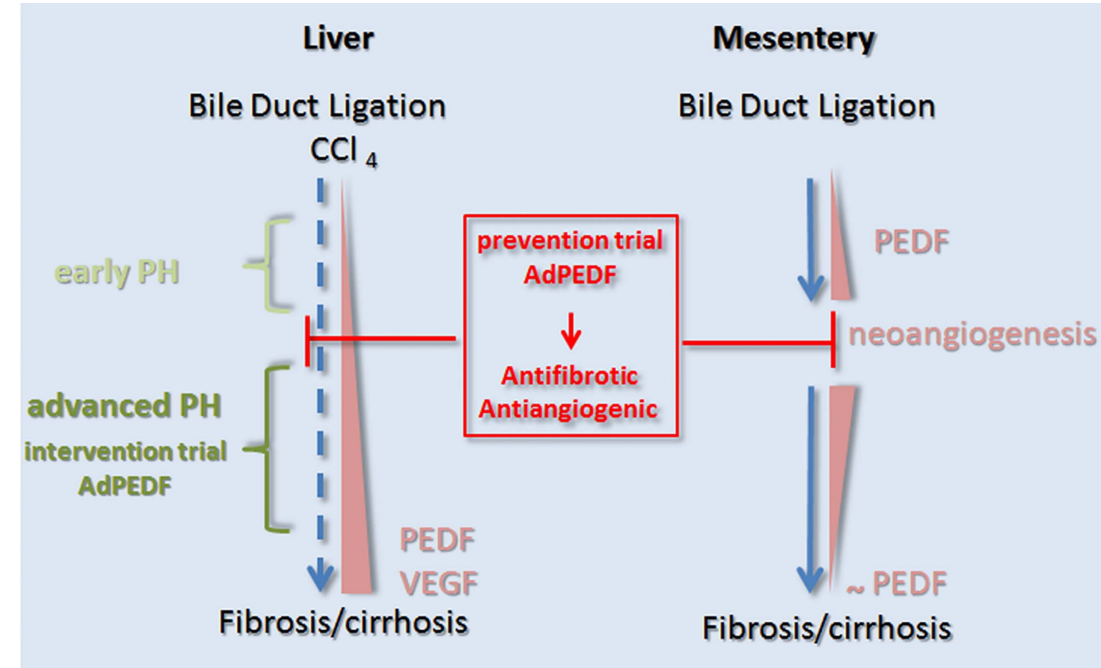

Figure 1 Pigment epithelium-derived factor (PEDF) gene transfer as a novel therapeutic agent. PEDF is overexpressed in the liver and mesenteric vascular bed in animal models of portal hypertension and cirrhosis. PEDF and vascular endothelial growth factor (VEGF) are unidirectionally upregulated and correlate with mesenteric neovascularisation and liver fibrogenesis during cirrhosis. In vivo PEDF gene transfer mediated by adenoviral vectors (AdPEDF) effectively suppresses mesenteric pathological angiogenesis and intrahepatic fibrogenesis in bile duct ligation $(\mathrm{BDL})$ rats, leading to a significant decrease in portal pressure. 
pressure from the subclinical to the clinically significant range would prevent many of the sequelae we observe.

Since neoangiogenesis actively contributes to PHT from the first steps of its development, ${ }^{1}$ drugs with antiangiogenic properties are attractive candidates to prevent PHT. $^{7}$ However, the development of clinical studies to investigate this has been hampered by drug toxicity and the concerns about interfering with physiological angiogenesis. ${ }^{7}$ The results presented by Mejias launches PEDF as a promising agent with the potential ability to affect portal pressure by specifically targeting pathological angiogenesis. Conversely, the significantly lower activity demonstrated by PEDF when given in a more advanced phase of liver disease raises some doubts concerning its potential to add something for the treatment of clinically significant PHT. However, since a modest effect on portal pressure is observed even in advanced cirrhosis, the combination of PEDF with vasoactive drugs deserves further experimental investigation, with the hope that working simultaneously on more therapeutic targets will maximise the impact on portal pressure.

In conclusion, the data presented in this study highlights again the importance of targeting VEGF-driven angiogenesis and reveals new important insights into the molecular working mechanism of PEDF as a possible antiangiogenic and antifibrotic agent in the treatment of early phase PHT. Hence, the next step should be trying to

translate these findings into clinical practice, where PEDF needs to confirm its capability to halt the rise of portal pressure, and to prove it can add something, eventually in association with or without non-selective $\beta$-blockers $(\mathrm{NS} \beta \mathrm{B})^{8}$ and/or anti-inflammatory agents, ${ }^{9}$ before clinical significant PHT has developed.

Competing interests None.

Provenance and peer review Commissioned; internally peer reviewed.

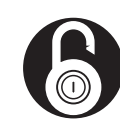

\section{OPEN ACCESS}

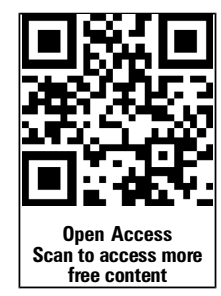

Open Access This is an Open Access article distributed in accordance with the Creative Commons Attribution Non Commercial (CC BY-NC 3.0) license, which permits others to distribute, remix, adapt, build upon this work non-commercially, and license their derivative works on different terms, provided the original work is properly cited and the use is noncommercial. See: http://creativecommons.org/licenses/ by-nc/3.0/

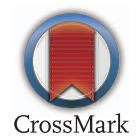

To cite Vespasiani-Gentilucci U, Rombouts K. Gut 2015;64:523-524.

Received 3 June 2014

Revised 7 June 2014

Accepted 9 June 2014
Published Online First 25 June 2014

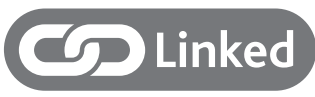

http://dx.doi.org/10.1136/gutjnl-2014-307138

Gut 2015;64:523-524.

doi:10.1136/gutjnl-2014-307583

\section{REFERENCES}

1 Garcia-Pagan JC, Gracia-Sancho J, Bosch J. Functional aspects on the pathophysiology of portal hypertension in cirrhosis. J Hepatol 2012;57:458-61.

2 Coulon S, Heindryckx F, Geerts A, et al. Angiogenesis in chronic liver disease and its complications. Liver Int 2011;31:146-62.

3 Mejias M. Antiangiogenic and antifibrotic activity of pigment epithelium-derived factor (PEDF) in bile duct-ligated portal hypertensive rats. Gut 2015;64: 657-66.

4 Craword SE, Fitchev P, Veliceasa D, et al. The many facets of PEDF in drug discovery and disease: a diamond in the rough or split personality disorder? Expert Opin Drug Discov 2013;8:769-92.

5 Manalo KB, Choong PF, Becerra SP, et al. Pigment epithelium-derived factor as an anticancer drug and new treatment methods following the discovery of its receptors: a patent perspective. Expert Opin Ther Pat 2011;21:121-30

6 Bosch J, Abraldes JG, Berzigotti A, et al. The clinical use of HVPG measurements in chronic liver disease. Nat Rev Gastroenterol Hepatol 2009;6:573-82.

7 Berzigotti A, Bosch J. Pharmacologic management of portal hypertension. Clin Liver Dis 2014;18:303-17.

8 Krag A, Wiest $R$, Albillos A, et al. The window hypothesis: haemodynamic and non-haemodynamic effects of beta-blockers improve survival of patients with cirrhosis during a window in the disease. Gut 2012;61:967-9.

9 Mehta G, Gustot T, Mookerjee RP, et al. Inflammation and portal hypertension-the undiscovered country. J Hepatol 18 Mar 2014. Epub ahead of print. 